\title{
In situ photocontrol of block copolymer morphology during dip-coating of thin films
}

\author{
Jaana Vapaavuori, Josué Grosrenaud, Christian Pellerin* and C. Geraldine Bazuin* \\ Département de chimie, Centre de recherche sur les matériaux auto-assemblés (CRMAA/CSACS), Université de Montréal, \\ C.P. 6128 Succ. Centre-ville, Montréal, QC, Canada H3C 3J7
}

\begin{abstract}
We demonstrate a unique combination of simultaneous top-down and bottom-up control of the morphology of block copolymer films by application of in situ optical irradiation during dip-coating. A light-addressable and blockselective small molecule, 4-butyl-4'-hydroxyazobenzene (BHAB), is introduced into a diblock copolymer of polystyrene and poly(4-vinylpyridine) (PS-P4VP) of $28.4 \mathrm{wt} \% \mathrm{P}_{4} \mathrm{VP}$ via supramolecular chemistry, notably by hydrogen bonding to $\mathrm{P}_{4} \mathrm{VP}$. We show that the spherical morphology of thin films dip-coated from a THF solution at slow withdrawal rates in the dark convert to cylindrical morphology when dip-coated under illumination. This is attributed to volume expansion of the $\mathrm{P}_{4} \mathrm{VP} / \mathrm{BHAB}$ phase due to trans-cis photoisomerization combined with a light-induced increase in BHAB uptake in the film. The demonstrated photocontrol highlights the potential of dip-coating as a scalable film preparation method that can be easily coupled with external stimuli to direct nanostructured self-assembly in the films as solvent evaporates.
\end{abstract}

Controlling the properties of soft materials at the nanoscale is paramount for high-technology applications in diverse domains. Many of these applications require nanostructured materials to be in the form of thin films of good surface quality, ${ }^{1}$ for example when used as nanolithographic templates for the electronics industry. ${ }^{2}$ Block copolymers (BCPs) are well suited for this purpose, given their propensity to self-assemble into well-defined morphologies, with typical periodicities in the $10-100 \mathrm{~nm}$ range, due to block immiscibility. ${ }^{3}$ In thin films, this leads to various surface patterns that are controllable at will by a number of parameters, both material (such as molecular weight, block volume fractions, interfacial interaction parameters, and addition of block selective components) and experimental (such as film preparation method, choice of solvent, and thermal/solvent annealing). ${ }^{1}$

In the present contribution, we couple the dip-coating film preparation technique with light as a remote external stimulus, to exert in situ optical control of self-assembly in supramolecular azo-containing BCPs. Dip-coating from BCP solutions leads to nanostructured films without the need for postprocessing such as thermal or solvent annealing to develop morphologies, since effective (if partial) solvent annealing occurs during solvent evaporation. ${ }^{4}$ This technique is commonly used in industry,6 and can be applied using minimal solution. ${ }^{7}$ Moreover, it is perfectly amenable to the simultaneous application of external stimuli, such as light, temperature and electric/magnetic fields, allowing combined bottom-up and top-down control of patterning in $\mathrm{BCP}$ thin films.

The capacity for precise temporal and spatial control makes light a particularly attractive mode of noncontact stimulus, which is often applied to azobenzene-based materials due to the photoisomerization of the azo moiety from the rodlike trans isomer to the more dipolar and bulky cis isomer. ${ }^{8-11}$ Intriguing examples of successful combinations of photoresponsiveness and block copolymers in solid-state films include reorienting the cylindrical morphology of BCPs through photoirradiation, ${ }^{12}$ con- trolling $3 \mathrm{D}$ cylindrical orientation through photoinduced surface patterning, ${ }^{13}$ and photoswitching the $2 \mathrm{D}$ morphology of Langmuir-Blodgett monolayers. ${ }^{14}$

As opposed to these studies done with covalently synthesized azo-containing BCPs, we take advantage here of a synthesis-free supramolecular approach as a highly versatile means to incorporate light-addressable components in BCPs. A commonly used supramolecular link is the hydrogen bond between phenol and pyridine moieties, ${ }^{15-17}$ including in the preparation of comb-like BCPs. ${ }^{18-20}$ Thus, commercially available diblock copolymers of polystyrene- $b$-poly(4-vinylpyridine) (PS-P4VP) combined with hydroxyl-functionalized azo molecules are a perfect starting point for explorations of in situ photocontrol of dipcoated film morphologies. Specifically, we chose a PS$\mathrm{P}_{4} \mathrm{VP}$ with $\mathrm{M}_{\mathrm{n}}(\mathrm{PS})=24 \mathrm{~kg} / \mathrm{mol}$ and $\mathrm{M}_{\mathrm{n}}\left(\mathrm{P}_{4} \mathrm{VP}\right)=9.5 \mathrm{~kg} / \mathrm{mol}$ and the azo molecule, 4-butyl-4'-hydroxy-azobenzene (BHAB), the molecular structures of which are shown in Figure 1a. BHAB was chosen due to its relatively high cis yield upon illumination and the sufficiently long half-life of $53 \mathrm{~min}$ for cis-BHAB in THF as compared to the timescale of the dip-coating experiments (withdrawing at the slowest dip-coating rate used took $16 \mathrm{~min}$ ). The dipcoating solvent is THF, the $\mathrm{BCP}$ solution concentration 10 $\mathrm{mg} / \mathrm{mL}$, and the molar ratio of $\mathrm{BHAB}$ to $\mathrm{VP}$ in solution is 0.25 . The system is designated as PS- $\mathrm{P}_{4} \mathrm{VP}(\mathrm{BHAB})_{0.25}$. The solution is not micellar as indicated by dynamic light scattering results.

The use of a supramolecular system in combination with dip-coating introduces additional control over film surface patterns and morphologies, as described in detail by us previously. ${ }^{4,21-23}$ Notably, we showed that the uptake of hydrogen-bonding small molecules (SM) in dip-coated PS-P4VP films varies with dip-coating withdrawal rate; in particular, for THF solutions, the molar SM/VP uptake ratio is low at very slow dip-coating rates and gradually increases to that in solution with increase in dip-coating rate. Since the $\mathrm{SM}$ is $\mathrm{P}_{4} \mathrm{VP}$-selective due to $\mathrm{SM}-\mathrm{VP}$ hydrogen bonding, this evolution in SM/VP uptake ratio im- 
plies that the effective volume fraction of the $\mathrm{P}_{4} \mathrm{VP}$-based phase in the drying film also increases with dip-coating rate, and was shown to be the main factor responsible for a morphology evolution in the dried films as a function of dip-coating rate. It must be emphasized that the final morphologies in the films are generally frozen-in and are not dry-film equilibrium morphologies. ${ }^{23}$ Film thickness also evolves with dip-coating rate in a V-shaped manner that defines the recently discovered "capillarity regime" (decreasing thickness vs. rate for slow rates) and the well known "draining regime" (increasing thickness vs. rate for fast rates), ${ }^{4-6}$ which can influence morphological orientation and film terracing. ${ }^{21,23-25}$ These variations highlight the fact that different thin film surface patterns can be obtained from a single supramolecular $\mathrm{BCP}$ solution composition simply by varying the dip-coating withdrawal rate. As shown below, coupling this with photocontrol further extends the possibilities of the technique.

In this context, it was reasoned that, if light irradiation causes photoisomerization of $\mathrm{P}_{4} \mathrm{VP}$-selective $\mathrm{BHAB}$, due to its more bulky cis form, it will change the volume fraction of the $\mathrm{P}_{4} \mathrm{VP}$-based phase and, if the system is sufficiently near a morphological phase boundary before immobilization sets in, dip-coating under illumination could result in a change in final film morphology compared to dip-coating in the dark. Although not anticipated, the uptake ratio of $\mathrm{BHAB}$, as will be shown below, increases under illumination and thus also contributes to increased volume fraction of the $\mathrm{P}_{4} \mathrm{VP}$-based phase.

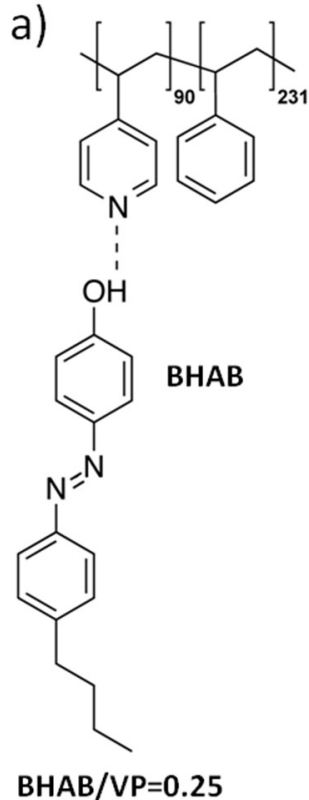

$\mathrm{BHAB} / \mathrm{VP}=0.25$

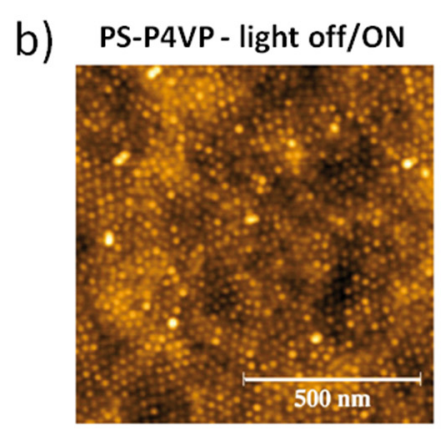

c)

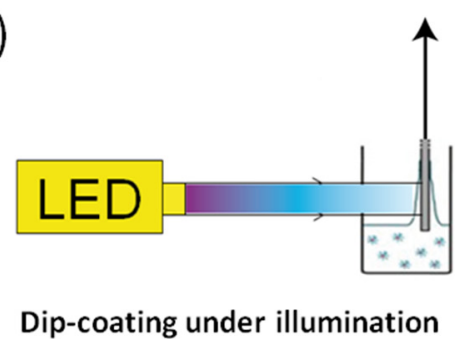

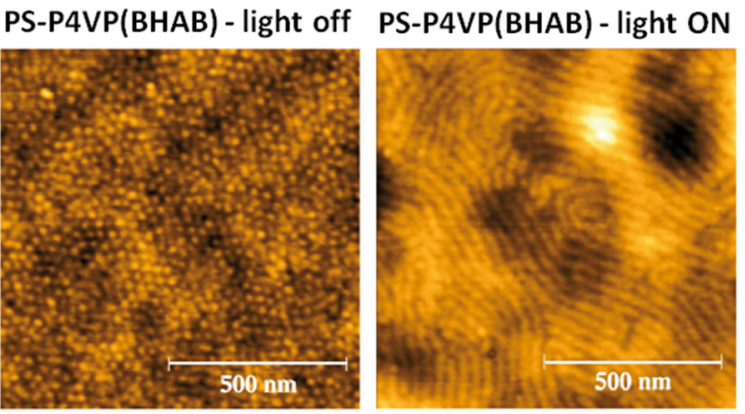

d)

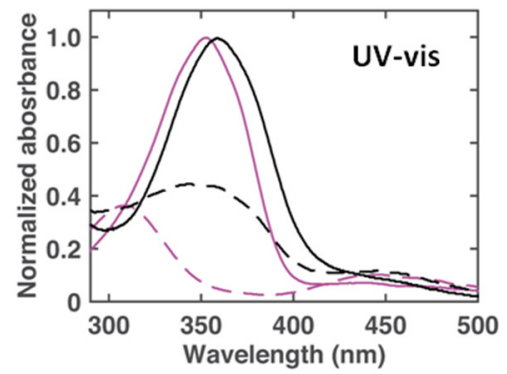

Figure 1. a) Structure of the supramolecular azo-containing BCP system studied: PS-P4VP and BHAB, where the molar ratio of $\mathrm{BHAB}$ to VP in the dip-coating THF solution is 0.25. b) Topographical AFM images (1X1 $\left.\mu \mathrm{m}^{2}\right)$ of thin films of PS$\mathrm{P}_{4} \mathrm{VP}$ (leftmost; z-scale $5 \mathrm{~nm}$ ) dip-coated in the dark (same pattern obtained under illumination), $\mathrm{PS}-\mathrm{P} 4 \mathrm{VP}(\mathrm{BHAB})$ (middle; z-scale $4 \mathrm{~nm}$ ), dip-coated in the dark, and PS-P4VP(BHAB) (rightmost; z-scale $8 \mathrm{~nm}$ ) dip-coated under $365 \mathrm{~nm}$ LED irradiation, all at a withdrawal rate of $0.5 \mathrm{~mm} / \mathrm{min}$ from THF solutions. c) Schematic diagram of the dip-coater coupled with an LED source. d) UV-visible spectra of a THF solution of PS-P4VP(BHAB) ${ }_{0.25}$ (magenta) and of a spin-coated PS$\mathrm{P}_{4} \mathrm{VP}(\mathrm{BHAB})_{0.25}$ thin film (black), before (solid lines) and directly after (dashed lines) irradiation.

To test the effect of photoirradiation on the film surface patterns or morphologies, the first step was to identify a dip-coating withdrawal rate region for the system under investigation where a transition in final film morphology occurs in the dark due simply to supramolecular control. Dip-coating from a BHAB-free block copolymer solution leads to only dot patterns (leftmost panel of Figure $1 b)$ as a function of dip-coating rate. Given the $\mathrm{P}_{4}$ VP fraction in the BCP (28.4 wt \%) and, in accordance with previous results ${ }^{4,21,23}$ using similar $\mathrm{P}_{4} \mathrm{VP}$ weight fractions, this pattern is associated with a spherical morphology composed of $\mathrm{P}_{4} \mathrm{VP}$ spheres embedded in a PS matrix. For PS- $\mathrm{P}_{4} \mathrm{VP}(\mathrm{BHAB})_{0.25}$, the same pattern of surface dots was observed in the film dip-coated at a withdrawal rate of $0.5 \mathrm{~mm} / \mathrm{min}$ (Figure $\mathrm{lb}$, middle panel). Since the BHAB/VP ratio is low in solution and even lower in the film (about 0.06 at this withdrawal rate; Figure $2 \mathrm{~b}$ ), the dot pattern must still reflect a spherical morphology (of $\mathrm{P}_{4} \mathrm{VP} / \mathrm{BHAB}$ dispersed in a PS matrix). It is followed by a mixed pattern of dots and stripes at $1.0 \mathrm{~mm} / \mathrm{min}$ and essentially only stripes at 2.0-10.0 $\mathrm{mm} / \mathrm{min}$, and is accompanied by an increase in BHAB uptake with dip-coating rate (Figure $2 \mathrm{~b}$ ). This pattern and the SM uptake evolution follows what we observed previously with other (smaller) hydrogen-bonding SMs using THF as the solvent,, ${ }^{4,22}$ where the stripes are as- 
sociated with a morphology of SM/P4VP cylinders oriented parallel to the substrate plane. Thus, there is a morphological transition in the vicinity of $1.0 \mathrm{~mm} / \mathrm{min}$ (where the two morphologies coexist) as a function of withdrawal rate for dip-coating in the dark that is driven by the increasing BHAB content taken up in the films with dip-coating rate.

Next, dip-coated films were prepared in the same withdrawal rate region, this time under in situ illumination by a $365-\mathrm{nm}$ LED light source $\left(40 \mathrm{~mW} / \mathrm{cm}^{2}\right)$, as depicted in Figure 1c. The LED wavelength is close to optimal for the system, since, as deduced from the residual trans absorption band under illumination (Figure 1d), it induces trans-cis photoisomerization of BHAB at a yield exceeding $90 \%$ in the $\mathrm{PS}-\mathrm{P}_{4} \mathrm{VP}(\mathrm{BHAB})_{0.25}$ solution (and also in a pure BHAB solution, not shown) and at least $57 \%$ in a dry spin-coated film (lower than in solution due to reduced mobility of the BHAB molecules in the solid state). Figure 1c (rightmost panel) shows that a stripe pattern, indicative of a horizontal cylindrical morphology, is obtained already at the lowest dipcoating rate used $(0.5 \mathrm{~mm} / \mathrm{min})$, and is maintained throughout the range investigated. [The periodicities of the patterns do not vary perceptibly with dip-coating rate or pattern type and they are all similar at 28, 30 and $32( \pm 2) \mathrm{nm}$ for the pure PS-P4VP and the PS$\mathrm{P}_{4} \mathrm{VP}(\mathrm{BHAB})_{0.25}$ dip-coated in the dark and under irradiation, respectively.] Clearly, in situ illumination has modified the morphology in the films dip-coated at 0.5 and $1.0 \mathrm{~mm} / \mathrm{min}$ from the $\mathrm{BHAB}$-containing solutions, and has presumably moved the morphology transition to lower dip-coating rates not tested here. (In contrast, no morphology change was observed in films dip-coated under illumination from the BHAB-free solution.)

While the photoinduced surface pattern change in the $\mathrm{PS}-\mathrm{P}_{4} \mathrm{VP}(\mathrm{BHAB})_{0.25}$ films might be attributed to an increase in the effective $\mathrm{P}_{4} \mathrm{VP} / \mathrm{BHAB}$ phase fraction due to the greater bulkiness and associated free volume of the cis isomer compared to the trans isomer, one may also ask if illumination modifies the BHAB uptake ratio. This question was addressed by infrared (IR) spectroscopy, where the $\mathrm{BHAB} / \mathrm{VP}$ uptake ratio was quantified with the aid of a calibration curve (Figure $\mathrm{S} 1$ ) using the relative intensities of the bands at 1505 and $1492 \mathrm{~cm}^{-1}$, attributed to BHAB and PS- $\mathrm{P}_{4} \mathrm{VP}$, respectively, and bearing in mind that hydrogen-bonding to $\mathrm{P}_{4} \mathrm{VP}$ does not affect the PS-P 4 VP absorption at $1492 \mathrm{~cm}^{-1} .{ }^{22}$ Since these bands overlap to some extent, as demonstrated in Figure 2a, a second derivative method was adopted to better resolve and quantify the contributions of these two bands. The results are given in Figure $2 \mathrm{~b}$, which shows that BHAB uptake under irradiation increases with dipcoating rate similarly to that in the dark, but, most importantly, the ratio is systematically higher for the irradiated samples than for the non-irradiated samples. It may be noted as well that the uptake ratio in the film dip-coated under illumination at $0.5 \mathrm{~mm} / \mathrm{min}$ and in the film dip-coated in the dark at $2 \mathrm{~mm} / \mathrm{min}$, both of which give a stripe pattern, are similar. The data in Figure $2 b$ thus imply that the morphological change at the rates of 0.5 and $1 \mathrm{~mm} / \mathrm{min}$ under illumination compared to in the dark must be attributed to the photoinduced uptake ratio increase in addition to the increased volume of the cis relative to trans isomer, both of these factors increasing the effective volume fraction of the $\mathrm{P}_{4} \mathrm{VP} / \mathrm{BHAB}$ phase during the $\mathrm{BCP}$ self-assembly process. We can speculate that the photoinduced BHAB uptake increase may be related to the change in dipole moment of $\mathrm{BHAB}$, which increases from 2.03 to $4.72 \mathrm{D}$ (as calculated by DFT) upon conversion from trans to cis, as is typical for azobenzenes. ${ }^{26}$ This can increase the electrostatic affinity between cis-BHAB and $\mathrm{P}_{4} \mathrm{VP}$ relative to cis-BHAB and THF, since 4-methylpyridine has a higher dipole moment than THF (2.70 vs. 1.75 ${ }^{27}$ ). Analogous changes in polymer-solvent interactions upon photoinduced polarity changes are responsible for significant cloud point temperature shifts in solutions of diblock copolymers possessing an azobenzene-containing block. ${ }^{28}$
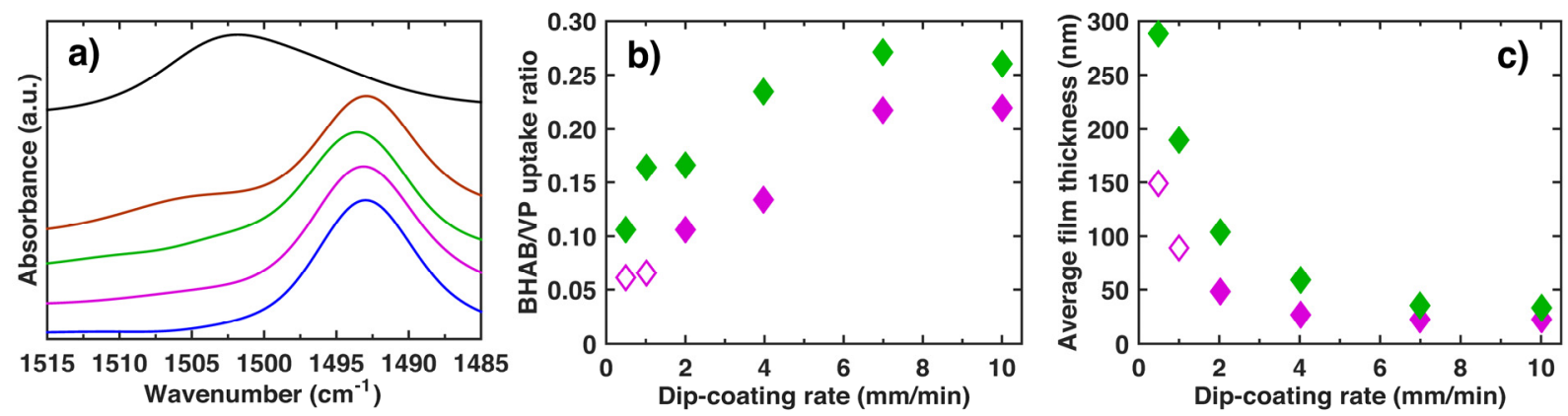

Figure 2. a) IR spectra of a drop-cast film of pure PS-P4VP (blue), films dip-coated at $0.5 \mathrm{~mm} / \mathrm{min}$ from a PS$\mathrm{P}_{4} \mathrm{VP}(\mathrm{BHAB})_{0.25}$ THF solution in the dark (magenta) and under illumination (green), a drop-cast film from the same solution (brown) and the melt-state spectrum of pure BHAB (black). b) BHAB/VP molar uptake ratio in, and c) average thickness of films dip-coated in darkness (magenta) and upon illumination (green) as a function of dip-coating withdrawal rate; open symbols indicate films with non-stripe patterns, closed symbols those with a stripe pattern. 
One may also wonder if film thickness, which, as mentioned above, varies with dip-coating rate, is affected by irradiation. Figure $2 \mathrm{c}$ shows that the thickness, measured by ellipsometry, decreases with dip-coating rate in the range investigated - as found previously, ${ }^{4,2123}$ for the slow dip-coating rate range involved, which corresponds to the capillarity regime - both in the dark and under irradiation. More interestingly, the irradiated samples are systematically thicker than the non-irradiated samples. The increase in thickness caused by irradiation was observed also for films dip-coated from pure (BHAB-free) solutions of PS-P4VP, as shown in Figure $\mathrm{S}_{2}$ (with no change in surface pattern, which remain in the form of dots). Since it is known that film thickness in the capillarity regime increases with temperature due to faster solvent evaporation, ${ }^{5}$ this photoinduced thickness increase is tentatively attributed to non-azo-related heating caused by the irradiation. In our case, this temperature increase is estimated to be about $10^{\circ} \mathrm{C}$, based on the observed photoinduced thickness increase (Figures $2 \mathrm{C}$ and $\mathrm{S} 2$ ), the temperature dependence of the THF vapor pressure, ${ }^{29}$ and the vapor pressure dependence of the film thickness in the capillarity regime. ${ }^{5}$ The photoinduced thickness or temperature increase is unlikely, however, to account for the photoinduced change in surface pattern from dots to stripes at the $0.5 \mathrm{~mm} / \mathrm{min}$ dip-coating rate. This is indicated in particular by the fact that the dot-to-stripe change observed in the dark as a function of dip-coating rate occurs in a thickness range of $150-50 \mathrm{~nm}$, with stripes observed in the 50-20 $\mathrm{nm}$ range, whereas the films dip-coated under irradiation, which all show stripes, range in thickness from about 300 to $30 \mathrm{~nm}$. Furthermore, when the PS$\mathrm{P}_{4} \mathrm{VP}(\mathrm{BHAB})_{0.25}$ solution is irradiated for 20 min prior to dip-coating followed immediately by dip-coating in the dark (giving a film with the same thickness as the one dip-coated in the dark from the non-irradiated solution), the film shows a mixed morphology of dots and stripes (Figure $\mathrm{S}_{3}$ ). This mixed morphology is attributed to partial relaxation of the cis isomers back to trans, resulting in a partially converted morphology and a measured $\mathrm{BHAB} / \mathrm{VP}$ uptake ratio that is intermediate to those measured in the dark and under irradiation.

Two last points may be raised. First, immobilization of the $\mathrm{BCP}$ matrix following solvent evaporation limits the mobility of the hydrogen-bonded azo molecules, as indicated by the decrease in photoisomerization yield in the solid-state film (Figure 1c), raising the question of whether or not cis-isomers can be trapped in the film. To address this, Figure za displays a selected region of the IR spectra of a drop of solution deposited on the ATR crystal, taken under in situ irradiation 10 min after its start and, again, 15 min after irradiation was interrupted, compared to the spectrum of a drop deposited with no irradiation. The irradiated drop (green spectrum) displays a prominent cis-BHAB band at $890 \mathrm{~cm}^{-1}$, along with the nearabsence of the trans band at $1138 \mathrm{~cm}^{-1}$. The spectrum (magenta) taken $15 \mathrm{~min}$ after cessation of irradiation is identical to that of the non-irradiated sample (black), showing that all cis-BHAB has relaxed thermally into trans-BHAB within the time elapsed. This is consistent with the fact that the half-life of the cis-BHAB is much shorter in these solid-state films than in THF solution, and the BCP environment thus tunes the kinetics of the photoreaction, as shown previously. ${ }^{30-33}$
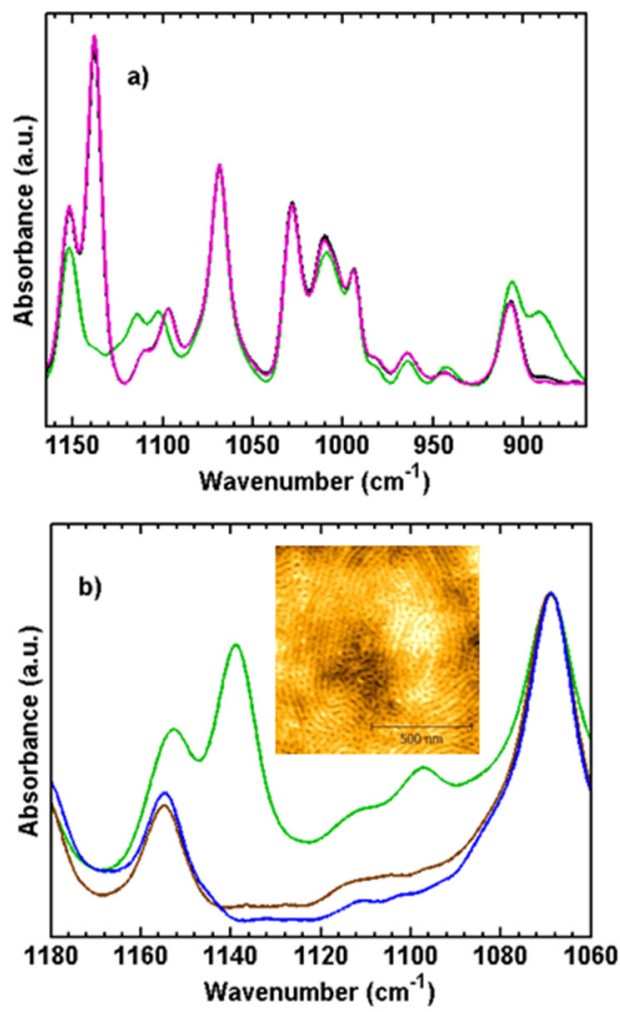

Figure 3. a) IR spectra of thin films of PS- $\mathrm{P}_{4} \mathrm{VP}(\mathrm{BHAB})_{0.25}$ drop-cast in darkness (black) and under $365 \mathrm{~nm}$ light (green), and the spectrum of the latter film (magenta) 15 min after turning off the illumination. The magenta curve essentially overlays the black curve. b) IR spectra of a drop-cast PS-P4VP film (blue) and a dip-coated PS$\mathrm{P}_{4} \mathrm{VP}(\mathrm{BHAB})_{0.25}$ film before (green) and after (brown) methanol rinsing. Inset: AFM height image of the methanol-rinsed film.

Secondly, it must be noted that the morphologies are stable once dip-coating is completed. In particular, it was not possible to induce a dot-to-stripe transition by illuminating a dried film that had been dip-coated in the dark at $0.5 \mathrm{~mm} / \mathrm{min}$. Furthermore, the BHAB molecules can be eliminated, after they have completed their function, by a selective solvent without changing the $\mathrm{BCP}$ morphology. This is illustrated in Figure $3 \mathrm{~b}$, which compares the spectra, before and after rinsing by methanol (a good solvent for $\mathrm{BHAB}$ and $\mathrm{P}_{4} \mathrm{VP}$ ), of a film that was irradiated during dip-coating at $0.5 \mathrm{~mm} / \mathrm{min}$ and of a film dip-coated from a BHAB-free solution. Clearly, the BHAB band at $1138 \mathrm{~cm}^{-1}$ is absent for the rinsed film, and the AFM image (inset in Figure $3 \mathrm{~b}$ ) shows the same stripe pattern as the irradiated BHAB-containing film in Figure $\mathrm{ib}$. This ability to selectively detach the photoactive moieties without influencing the overall morphology allows the production of optically transparent films and, in gen- 
eral, serves as a further demonstration of the flexibility provided by the supramolecular approach as compared to conventional synthesis of functional block copolymers.

The results presented demonstrate, in a proof-of-concept manner, that the morphology of BCP films containing block-selective, supramolecularly attached azobenzene units can be controlled by light during dip-coating. This method of in situ optical switching of block copolymer nanostructures during film processing is a cost-effective and easily scalable preparation method for good optical quality thin films with self-assembled nanostructures, without necessitating further post-processing. It is also highly versatile, since, in principle, a desired morphological transition can be displaced at will by astute combinations of the choice of solvent, amount and choice of the azo or other photoresponsive SM in the solution, and relative block fractions, molecular weight and chemical composition of the BCP. Additionally, the nanostructures are stable, allowing removal of the supramolecularly attached molecules by a selective solvent and thus enabling applications requiring optically transparent films. This original combination of simultaneous bottom-up and topdown control of nanostructured materials is further amenable to masking techniques for producing novel complex patterns, where, for example, one type of pattern is obtained in the masked regions and another type in the exposed regions of the film.

\section{ASSOCIATED CONTENT}

\section{Supporting Information}

Experimental details and additional figures. The Supporting Information is available free of charge via the Internet at http://pubs.acs.org.

\section{AUTHOR INFORMATION}

\section{Corresponding Authors \\ *Email: geraldine.bazuin@umontreal.ca \\ *Email: c.pellerin@umontreal.ca}

\section{Notes}

The authors declare no competing financial interest.

\section{ACKNOWLEDGMENTS}

The research was funded by the Natural Sciences and Engineering Research Council (NSERC) of Canada and the Fonds de Recherche du Québec - Nature et Technologies (FRQNT). J.V. is grateful to Emil Aaltonen Foundation, Finnish Cultural Foundation, and FRQNT for postdoctoral grants. We thank Calcul Québec and Compute Canada for access to the Mammoth supercomputer.

\section{REFERENCES}

1. Li, M.; Coenjarts, C.A.; Ober, C.K. Adv. Polym. Sci. 2005, 190, $183-226$.

2. Galatsis, K.; Wang, K.L.; Ozkan, M.; Ozkan, C.S.; Huang, Y.; Chang, J.P.; Monbouquette, H.G.; Chen, Y.; Nealey, P.; Botros, Y. Adv. Mater. 2010, 22, 769-778.

3. Bates, F.S.; Fredrickson, G.H. Physics Today 1999, 52, 32.

4. Roland, S.; Prud'homme, R.E.; Bazuin, C.G. ACS Macro Letters 2012, 1, 973-976.

5. Faustini, M.; Louis, B.; Albouy, P.A.; Kuemmel, M.; Grosso, D. J. Phys. Chem. C 2010, 114, 7637-7645.

6. Grosso, D. J. Mater. Chem. 2011, 21, 17033-17038.

7. Ceratti, D.R.; Louis, B.; Paquez, X.; Faustini, M.; Grosso, D. Adv. Mater. 2015, 27, 4958-4962.

8. Lee, S.; Kang, H.S.; Park, J.K. Adv. Mater. 2012, 24, 2069-2103.

9. Mahimwalla, Z.; Yager, K.G.; Mamiya, J.-i.; Shishido, A.; Priimagi, A.; Barrett, C.J. Polym. Bull. 2012, 69, 967-1006.

10. Natansohn, A.; Rochon, P. Chem. Rev. 2002, 102, 4139-4176.

11. Seki, T.; Nagano, S.; Hara, M. Polymer 2013, 54, 6053-6072.

12. Yu, H.; Iyoda, T.; Ikeda, T. J. Am. Chem. Soc. 2006, 128, 1101011011.

13. Morikawa, Y.; Kondo, T.; Nagano, S.; Seki, T. Chem. Mat. 2007, 19, 1540-1542.

14. Kadota, S.; Aoki, K.; Nagano, S.; Seki, T. J. Am. Chem. Soc. 2005, 127, 8266-8267.

15. Ikkala, O.; ten Brinke, G. Science 2002, 295, 2407-2409.

16. Sallenave, X.; Bazuin, C.G. Macromolecules 2007, 40, 53265336.

17. Vapaavuori, J.; Valtavirta, V.; Alasaarela, T.; Mamiya, J.-I.; Priimagi, A.; Shishido, A.; Kaivola, M. J. Mater. Chem. 2o11, 21, 15437-15441.

18. Bondzic, S.; de Wit, J.; Polushkin, E.; Schouten, A.J.; ten Brinke, G.; Ruokolainen, J.; Ikkala, O.; Dolbnya, I.; Bras, W. Macromolecules 2004, 37, 9517-9524.

19. Cui, L.; Dahmane, S.; Tong, X.; Zhu, L.; Zhao, Y. Macromolecules 2005, 38, 2076-2084.

2o. van Zoelen, W.; Asumaa, T.; Ruokolainen, J.; Ikkala, O.; ten Brinke, G. Macromolecules 2008, 41, 3199-3208.

21. Roland, S.; Gaspard, D.; Prud'homme, R.E.; Bazuin, C.G. Macromolecules 2012, 45, 5463-5476.

22. Roland, S.; Pellerin, C.; Bazuin, C.G.; Prud'homme, R.E. Macromolecules 2012, 45, 7964-7972.

23. Roland, S.; Gamys, C.G.; Grosrenaud, J.; Boissé, S.; Pellerin, C.; Prud'homme, R.E.; Bazuin, C.G. Macromolecules 2015, 48, $4823-4834$.

24. Morikawa, Y.; Nagano, S.; Watanabe, K.; Kamata, K.; Iyoda, T.; Seki, T. Adv. Mater. 20o6, 18, 883-886.

25. Phillip, W.A.; Hillmyer, M.A.; Cussler, E.L. Macromolecules 2010, 43, 7763-7770.

26. Hartley, G. Nature 1937, 140, 281.

27. CRC Handbook of Chemistry and Physics, 76th ed.; CRC Press, Inc.: Boca Raton. FL, USA, 1995;

28. He, J.; Tremblay, L.; Lacelle, S.; Zhao, Y. Polym. Chem. 2014, 5, 5403-5411.

29. Scott, D.W. J. Chem. Thermodyn. 1970, 2, 833-837.

30. Frenz, C.; Fuchs, A.; Schmidt, H.-W.; Theissen, U.; Haarer, D. Macromol. Chem. Phys. 2004, 205, 1246-1258.

31. Moriya, K.; Seki, T.; Nakagawa, M.; Mao, G.; Ober, C.K. Macromol. Rapid Commun. 20oo, 21, 1309-1312.

32. Tsao, S.-C.; Lo, C.-T. RSC Advances 2014, 4, 23585-23594.

33. Wei, R.; He, Y.; Wang, X. RSC Advances 2014, 4, 5838658396. 
For Table of Contents use only:

In situ photocontrol of block copolymer morphology during dip-coating of thin films

Jaana Vapaavuori, Josué Grosrenaud, Christian Pellerin* and C. Geraldine Bazuin*

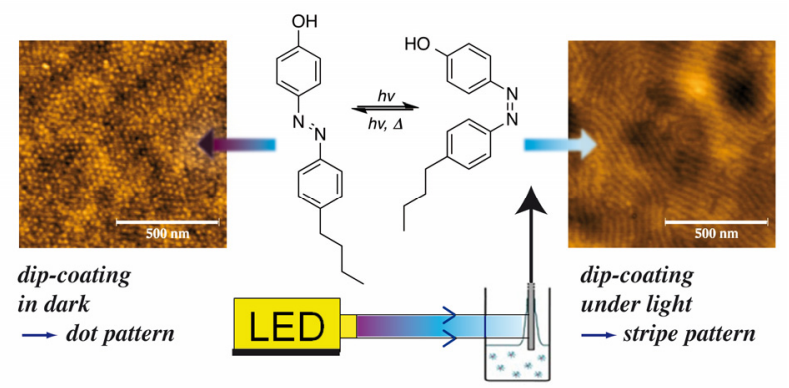

